\title{
Gradhiva
}

GRADHIV

Revue d'anthropologie et d'histoire des arts

16 | 2012

Chines, l'État au musée

\section{Gilbert Rouget et la mission Ogooué-Congo (1946). Institution et épistémologie dans l'histoire de l'ethnomusicologie en France}

Gilbert Rouget and the Ogooué-Congo Expedition (1946). Institution and

Epistemology in the History of French Ethnomusicology

\section{Brice Gérard}

\section{OpenEdition \\ Journals}

Édition électronique

URL : http://journals.openedition.org/gradhiva/2538

DOI : 10.4000/gradhiva. 2538

ISSN : 1760-849X

\section{Éditeur}

Musée du quai Branly Jacques Chirac

Édition imprimée

Date de publication : 1 décembre 2012

Pagination : 192-215

ISBN : 978-2-35744-048-7

ISSN : 0764-8928

\section{Référence électronique}

Brice Gérard, "Gilbert Rouget et la mission Ogooué-Congo (1946). Institution et épistémologie dans I'histoire de l'ethnomusicologie en France », Gradhiva [En ligne], 16 | 2012, mis en ligne le 01 décembre 2015, consulté le 10 décembre 2020. URL : http://journals.openedition.org/gradhiva/2538; DOI : https://doi.org/10.4000/gradhiva.2538 


\section{Gilbert Rouget et la mission Ogooué-Congo (1946)}

Institution et épistémologie dans l'histoire de l'ethnomusicologie en France

par Brice Gérard

Gilbert Rouget, ethnomusicologue né en 1916 et assistant d'André Schaeffner au musée de l'Homme depuis 1942, a participé à la mission Ogooué-Congo en 1946. L'analyse de plusieurs documents inédits, en particulier ses notes de terrain, et le contenu de différents entretiens permettent de décrire les contours épistémologiques d'une articulation spécifique entre ethnographie et utilisation des techniques d'enregistrement sonore, de manière à situer plus globalement la mission Ogooué-Congo dans l'histoire de l'ethnomusicologie, domaine de savoir alors en cours d'institutionnalisation en France. 
Gilbert Rouget, ethnomusicologue né en 1916 et assistant d'André Schaeffner au musée de l'Homme depuis 1942, a participé de juillet à décembre 1946 à la mission Ogooué-Congo, qui a parcouru depuis Brazzaville diverses régions des colonies françaises du Moyen-Congo (aujourd'hui république du Congo) et du Gabon. Ses membres ont notamment séjourné à proximité des pygmées babinga pendant six semaines, avant de gagner le Gabon et de descendre le fleuve Ogooué de Franceville à Lambaréné $\mathbf{1}$.

Quelques témoignages écrits, principalement ceux de Noël Ballif, l'organisateur de la mission, permettent de découvrir différents épisodes de cette expédition pluridisciplinaire, au sein de laquelle Rouget était le seul spécialiste de la musique (Ballif 1954, 1992; Hartweg 1961). Je souhaite évaluer, en me concentrant sur son activité, l'importance de la mission Ogooué-Congo dans I'histoire de l'ethnomusicologie en France et contribuer ainsi à l'histoire de cette discipline ${ }^{2}$.

Dans l'ouvrage collectif intitulé Qu'est-ce qu'une discipline? (Boutier, Passeron et Revel 2006), différentes contributions permettent de retenir, comme point de départ d'une réflexion sur la notion de discipline, trois dimensions qui lui sont attachées: les dimensions institutionnelle, pédagogique et, de façon plus problématique, la dimension partagée du collectif. Sur ce point, Jean-Louis Fabiani propose en particulier une relecture de l'œuvre de Thomas Kuhn en s'arrêtant sur les notions de paradigme et de matrice disciplinaire, puis rend compte de certains travaux de JeanClaude Passeron pour qui le régime des sciences sociales relève davantage de la pluralité théorique, c'est-à-dire de la «coexistence plus ou moins pacifique de plusieurs formes d'articulation entre des collectifs et des objets » (Fabiani 2006: 22).

La mission Ogooué-Congo s'inscrit clairement dans un processus d'institutionnalisation inauguré en 1929, quand Schaeffner entre au musée d'Ethnographie du Trocadéro (qui deviendra le musée de l'Homme en 1937) pour s'occuper des instruments de musique présents dans les collections et créer à cette occasion un service d'organologie, appelé par la suite département d'Ethnologie musicale (1933), puis département d'Ethnomusicologie (1954). Schaeffner fonde en particulier en 1932, au sein du même département, une phonothèque dont les collections se sont progressivement enrichies. Mes recherches m'incitent par ailleurs à considérer qu'une première période dans ce processus d'institutionnalisation s'achève en 1961, quand la VIe section (sciences sociales) de l'École pratique des hautes études, sur une proposition de Claude Lévi-Strauss, organise le premier enseignement spécialisé en ethnomusicologie. Celle-ci semble alors pleinement constituée en discipline sur les plans institutionnel et pédagogique; mais une discipline ne se réduit pas à ces deux dimensions. Les réflexions sur la dimension partagée du collectif sont indissociables d'une interrogation plus globale sur l'articulation entre les identités épistémologiques et les frontières institutionnelles, dans le sillage du livre de Passeron Le Raisonnement sociologique. Un espace non poppérien de l'argumentation (2006 [1991]), qui démontre l'indiscernabilité épistémologique de l'anthropologie, de la sociologie et de l'histoire. 
Différentes sources inédites permettent précisément d'étudier et de comprendre l'épistémologie engagée dans les travaux de Rouget en mission en 1946, au premier rang desquelles ses notes de terrain. II faut souligner dans cette perspective l'apport décisif de recherches qui, dans le cadre de l'histoire de l'anthropologie, ont accordé toute leur importance aux notes de terrain des ethnologues, d'abord en contexte anglo-saxon (Sanjek [éd.] 1990), puis en France depuis une journée d'étude organisée par Marion Abélès et Marie-Dominique Mouton en 1999 et prolongée par un dossier de Gradhiva intitulé «Archives et anthropologie » (Jamin et Zonabend [dir.] 2001-2002).

Dans quelle mesure l'évocation précise de la mission Ogooué-Congo, replacée dans son contexte mais analysée en détail à partir de son propre protocole méthodologique, permet-elle de situer et de caractériser ce moment dans l'histoire d'un domaine de savoir en cours d'institutionnalisation?

\section{Institution et objectifs}

Les activités de Rouget au musée de l'Homme, dans les quelques années qui précèdent son départ pour la mission Ogooué-Congo, sont directement liées au développement de la phonothèque. Dans une lettre de juillet 1942, Henri Victor Vallois, qui dirige alors l'institution à la place de Paul Rivet, exilé en Colombie, précise par exemple que «M. Rouget a poursuivi l'établissement des fiches descriptives et l'analyse des fonds de disques d'Afrique (Soudan, Mauritanie, Somalies, Mozambique), et a commencé les fiches descriptives des collections d'instruments de musique: série de hautbois ${ }^{3}$ ". À l'automne 1945, deux courriers de Thérèse Rivière, dont l'un est adressé à Germaine Tillion, montrent qu'il a été chargé d'effectuer, en vue d'une exposition temporaire sur l'Aurès, la gravure sur disque des cylindres enregistrés dix ans plus tôt par les deux jeunes femmes en mission dans cette région 4 .

Rouget a lui-même évoqué le caractère formateur de cet ensemble d'activités. II a précisé dans un témoignage audiovisuel récent que Rivet, revenu d'Amérique latine à la Libération et devenu président de la Radiodiffusion française, avait consulté Schaeffner au sujet d'une grande expédition de prise de son à Madagascar en 1939, dont toute la musique gravée se trouvait stockée à la Radiodiffusion. Schaeffner avait suggéré à Rivet une édition sur disque, puis était lui-même parti en mission en Afrique après avoir chargé Rouget de «faire le job». Ce dernier, en utilisant les notes «très intéressantes" prises par Schaeffner, réalisa ainsi sa première édition (30 disques, 60 faces). II ajoute dans le même témoignage: «Sur quoi je suis parti à Ogooué-Congo et bien entendu je me suis dit: je vais faire la même chose. » (Jourdain 2008)

Deux documents d'archive permettent de comprendre plus précisément ses objectifs à la veille de son départ en Afrique. II s'agit d'abord de la demande par Rivet d'un ordre de mission pour Rouget et Raoul Hartweg 5 (fig. 1). Le passage consacré au premier insiste sur l'importance de la collecte d'enregistrements sonores (500 faces de disque).
1. Je remercie vivement Gilbert Rouget d'avoir accepté de s'entretenir, parfois longuement, sur différents sujets et de m'avoir prêté plusieurs documents précieux, en particulier ses notes de terrain. Je remercie par ailleurs Esteban Buch et Bernard Lortat-Jacob pour leur lecture et leurs commentaires attentifs de ce texte.

2. Les travaux dans ce domaine sont rares et anciens. Un dossie constitué par Charles Boilès et Jean-Jacques Nattiez (1977) est souvent cité dans des synthèses plus récentes, par exemple dans la partie historique du Précis d'ethnomusicologie de Simha Arom et Frank Alvarez-Péreyre (2007). Une bibliographie plus abondante permet d'inscrire la musique dans une réflexion élargie sur l'histoire des sciences humaines, comme l'illustre un numéro de la Revue d'histoire des sciences humaines (Campos, Donin et Keck [éd.] 2006), dont une partie est consacrée à Schaeffner. Pour une mise en perspective récente de l'épistémologie de Schaeffner dans l'histoire de l'ethnomusicologie, voir Gérard 2009.
3. Archives du musée de l'Homme (désormais AMH), bibliothèque du Muséum national d'histoire naturelle, Paris, 2 AM 1 A13b.

4. $\mathrm{AMH}, 2 \mathrm{AM} 1 \mathrm{~A} 14 \mathrm{C}$.

5. $\mathrm{AMH}, 2 \mathrm{AM} 11 \mathrm{D} 4 \mathrm{C}$ 
Mon cher Ami,

Je vous serals très reconnaissent de bien vouloir accorder une Mission a Hessieurs ROUGES et HARTWEG, aldes-techniques au liusée de I'Homme, devant partir en Afrique Equatoriale lo I7 juiliet prochain.

Au cours de cette Mission, thonsieur irouget recueiliera des documents musicaux et linguistiques de promidre importanco. Il compte ramener en particu $110 r, 500$ faces de dieques constituant autant de documents différonts concernant des populations dont on an possède à $I^{\prime}$ haure actuelle aucun eniegistrement. Il enregistrera égalemont des documents lingui tiques et collestera les instruments de musique des populations étudiées.

De son câté, Monsleur Hartweg effectuera des recherches anthropologiques, ( mensurations, 6tudes hómatologiques) ot recueillera près dos services Sanitalres des pièces opératolres ou d'autopsie, en vue d'études d'histolog10 comparte des races humaines. Les efforts de liessieurs Rouget et Hartweg méritent d'ôtre grandement soutenus. Bspérant que vous leur donnerez satisfaction, jo vous prio de crolre, mon cher Ami, à mes sentiments affectueusement dévoús.

Monsleux A. URBAIN

D1recteur du liusérm

57 rue Cuvier

PARIS 50 
L'autre document est plus précis. II s'agit d'un feuillet dont le texte n'est ni daté, ni signé, et qui se trouve aujourd'hui parmi les archives du musée de l'Homme, à l'intérieur d'une chemise blanche intitulée Département d'ethnologie musicale ${ }^{6}$ (fig. 2).

Deux éléments autorisent l'utilisation de ce dernier document. D'une part, le contenu fait directement référence à la mission (personnes nommées, objectifs de chacun, etc.) et a été nécessairement rédigé en 1946 (à partir du moment où est conçu le projet de l'expédition). L'origine et la forme de l'archive révèlent d'autre part clairement qu'il s'agit d'un document officiel dont Rivet, si l'on compare précisément les deux textes, s'est manifestement inspiré pour rédiger sa demande d'ordre de mission, et qui formule les objectifs des travaux envisagés sur le terrain ${ }^{7}$. Le passage consacré à Rouget explicite différentes tâches déjà évoquées, mais contient surtout un véritable questionnaire destiné à orienter la rédaction des fiches accompagnant les enregistrements.

Ce questionnaire rappelle premièrement des documents produits auparavant au sein de l'institution, au premier rang desquels les Instructions sommaires pour les collecteurs d'objets ethnographiques (1931), rédigées à la veille de la mission Dakar-Djibouti et proposant «un système de classement et un modèle de fiche descriptive des objets ethnographiques qui, jusqu'à une date récente, étaient toujours utilisés pour cataloguer et étudier les collections entrant au musée de l'Homme» (Jamin 1996: 30). Ce précédent illustre a sans doute constitué une source d'inspiration, mais le questionnaire de 1946 se distingue par deux spécificités relatives. Les enregistrements sonores, plus que les instruments de musique, acquièrent pleinement le statut d'objets qu'il faut documenter par une fiche descriptive. Par ailleurs, ce questionnaire est à usage interne, explicitement destiné à guider le travail de terrain de Rouget, alors que les Instructions de 1931, tirées à plusieurs milliers d'exemplaires, «devaient être diffusées auprès des administrateurs et colons résidant dans les territoires que traverserait la mission au cours de son périple, afin que les objets recueillis au hasard de leurs tournées ou de leurs activités soient désormais accompagnés d'une masse de renseignements normalisés qui permettent aisément de les identifier, authentifier et répertorier » (ibid. : 28-30).

Un tel questionnaire présente deuxièmement un intérêt certain pour I'historien d'une discipline. La prise de notes ethnographiques, sans doute réalisée en interaction constante avec la réalité observée, n'en est pas moins orientée par un programme de travail défini en amont. Ce document contient un ensemble de prescriptions méthodologiques qu'on peut lire dans cette perspective et en sollicitant surtout sa vertu heuristique. II apparaît comme un modèle, non pas au sens d'un ensemble de règles déterminant nécessairement et exclusivement le travail de terrain, mais en tant que formalisation a priori de ce qui est attendu comme type d'observation.

Dans la partie du texte qui concerne la musique, il est ainsi précisé que la fiche rédigée pour chaque document sonore doit porter «le nom du lieu, la race, la langue, le sexe et le nombre des exécutants, leur position sociale, les instruments employés (et leurs noms respectifs), le sujet
6. $\mathrm{AMH}, 2 \mathrm{AM} 11 \mathrm{l} 1 \mathrm{~b}$ Ce dossier contient des documents d'époques différentes, tous issus du département fondé par Schaeffner et pour la plupart très précisément identifiés.

\section{ci-contre}

fig. 1

Demande d'un ordre de mission par Paul Rivet.

\section{ci-dessous}

fig. 2

«Enquête d'ethnographie musicale et de linguistique"

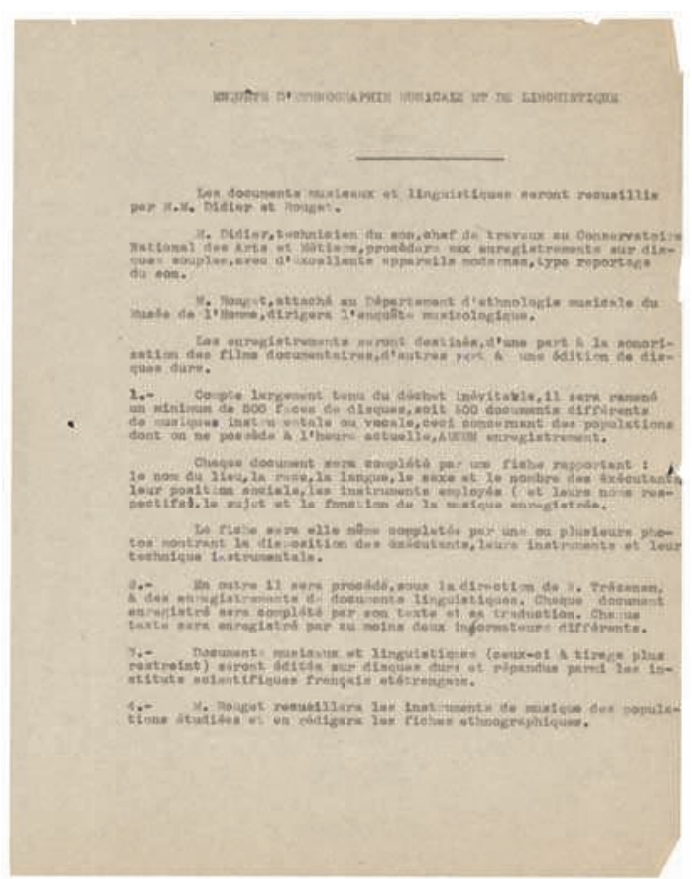

7. L'identification précise du document reste

problématique.

Faisant l'hypothèse que

son auteur était Schaeffner, qui livrait ainsi le programme de travail relevant de son département, j'en ai montré une copie à Gilbert Rouget pour lui demander son souvenir ou son sentiment: le texte lui a semblé «trop sec, trop direct pour être du Schaeffner », qui par André Didier et « qui n'avait pas à faire ce genre de choses" pour celui qui était alors son assistant et son secrétaire.

Rouget pense plutôt avoir écrit ces lignes lui-même, à la demande de Schaeffner qui les aurait ensuite transmises à Rivet (entretien, 5 juin 2009). cependant ailleurs ne savait pas qui était 
8. Entretien, 11 octobre 2009.

9. "Cahier ETHNO"

I: 5-12 août 1946 :

II: 13-17 août;

III : 18 août- 5 septembre;

IV: 6-11 septembre;

V: 10-17 septembre;

VI: 18-25 septembre;

VII: 26 septembre-3 octobre;

VII bis : $3-11$ octobre;

VIII : 28 octobre-fin novembre.

10. Gilbert Rouget, Notes de terrain, "Avertissement"

i. L'ensemble du volume

commente les faces

de disque 5 à 651 . II

faut souligner que la

dactylographie réalisée a

posteriori à partir des notes

originales n'a pas donné lieu

à une réorganisation générale

du matériau, comme l'indique

ce passage de l'avertissement

(ii): " Ces notes [originales]

ont été prises sur huit carnets

de format oblong $21 \times 13$

papier quadrillé et à feuilles

détachables portant les

numéros «ETHNO» I, II, III,

IV, V, VI, VII, VIII. Le numéro

VII bis correspond non pas à

un carnet mais à des feuilles

de cahier. Le carnet numéro

VIII est celui qui correspond

à la descente de l'Ogooué

et commence à Franceville.

Quelques feuilles volantes

[...] ont été dactylographiées

et incorporées aux dates

correspondantes." et la fonction de la musique enregistrée ». Le contenu de cette fiche est donc strictement ethnographique et n'encourage pas explicitement, par exemple, une enquête sur la structure du langage musical ou sur l'historicité des faits musicaux.

\section{Observations et informateurs}

Interrogé au cours d'un entretien récent sur les débuts de sa carrière, Rouget a écarté l'emploi du terme «épistémologie» pour évoquer la mission Ogooué-Congo («le mot est trop fort»), considérant qu'il était «alors bien jeune » et expliquant qu'il avait reçu à ce moment des «directives d'ethnographie classique (recueillir des faits avant tout, pas de dimension théorique, et sur ce plan je n'ai pas changé) ". Selon lui, on ne peut parler d'épistémologie que pour des périodes ultérieures de sa carrière, par exemple au sujet de son livre La Musique et la Transe (Rouget 1980). II a ensuite défini plus globalement sa conception de la discipline: une ethnomusicologie "substantielle» (le mot est revenu plusieurs fois au cours de l'entretien), "descriptive", "destinée à conserver, à contribuer à la sauvegarde" des faits recueillis; d'où un rejet des «belles théories", par exemple sur les gammes ou les échelles musicales. II a alors nuancé ses propos en évoquant les travaux de Constantin Brăiloiu, qui l'ont passionné, tout en ajoutant: "Mais même Brăiloiu était beaucoup dans la collecte, etc." Rouget a conclu en expliquant qu'il n'avait jamais aimé la théorie en ethnomusicologie, ce qu'illustrent selon lui ses travaux réalisés au cours de la mission Ogooué-Congo ${ }^{8}$.

L'analyse des notes de terrain issues de cette mission permet d'illustrer et de préciser ces affirmations générales et récentes. Ce document constitue un volume de 289 pages dactylographiées, divisé en neuf "cahiers" et organisé de façon strictement chronologique, en suivant la progression de la mission 9 . Pour chaque cahier, le texte se présente globalement comme une succession de notes (de quelques lignes à quelques pages chacune) précédées d'un numéro indiquant la face d'un disque gravé sur le terrain. Les premières lignes de l'avertissement rédigé par l'auteur en début de volume justifient cette structure globale:
Ces notes ont été dactylographiées à partir des carnets de notes que j'ai écrits au cours de la mission Ogooué-Congo et qui étaient destinés principalement à constituer la documentation concernant les enregistrements sur disque ${ }^{10}$.
Pour analyser ce document, on observera avec profit la manière dont Rouget a mis en pratique les prescriptions formulées dans le feuillet issu du département d'Ethnologie musicale et présenté plus haut comme un modèle. Un grand nombre de renseignements attendus dans ce dernier document sont effectivement indiqués par l'auteur dans ses notes de terrain: il rapporte systématiquement le nom du lieu et la «race» du ou des musiciens, ainsi que les instruments employés. II mentionne également, régulièrement, le sexe et le nombre d'exécutants.

L'organisation chronologique des cahiers explique que toutes les faces de disque soient contextualisées par la date, le lieu et la race: on trouve ces éléments dans les sommaires de chaque cahier (fig. 3 ; Fort-Rousset était une ville du Congo, Kuyu désigne un nom de peuple) et dans le cours 


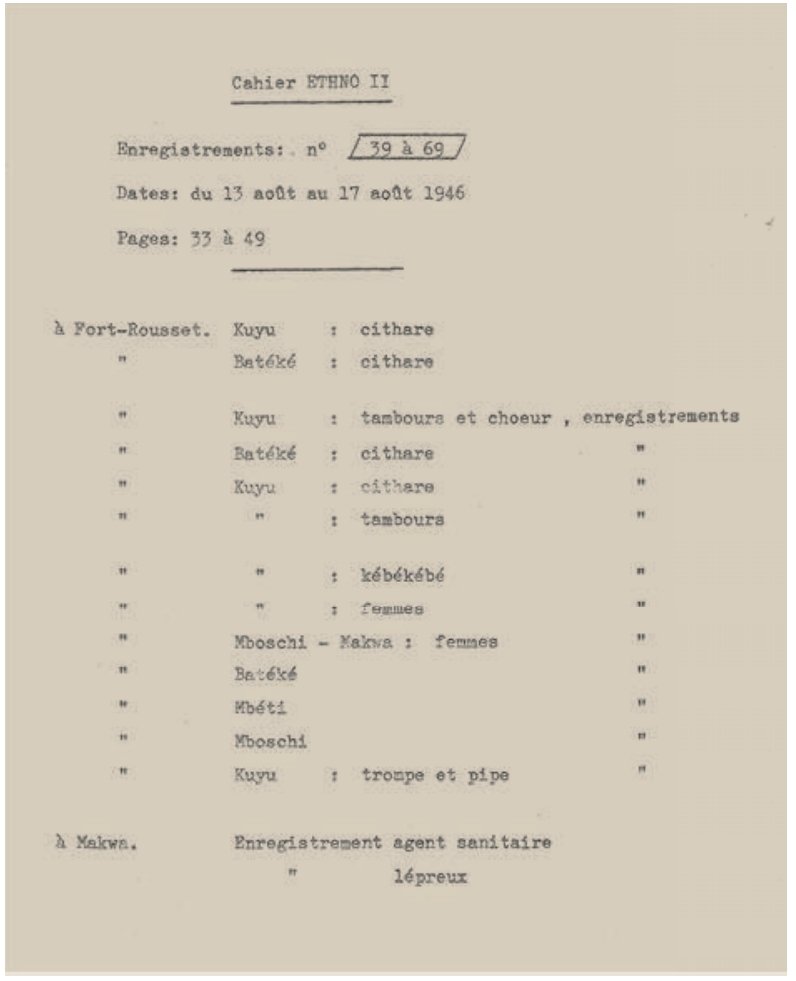

fig. 3 Sommaire du cahier

«ETHNO» II, Notes de terrain: 33.

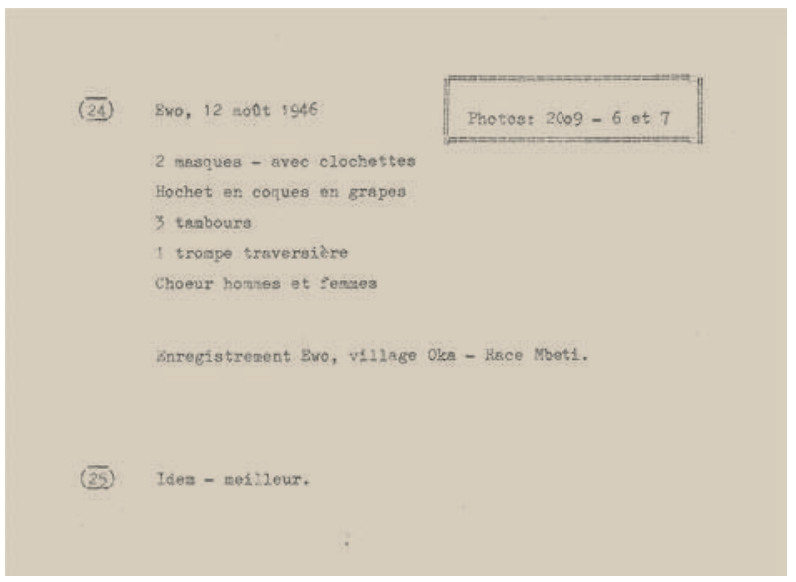

fig. 4 Faces de disque 24 et 25 ,

Notes de terrain : 25.

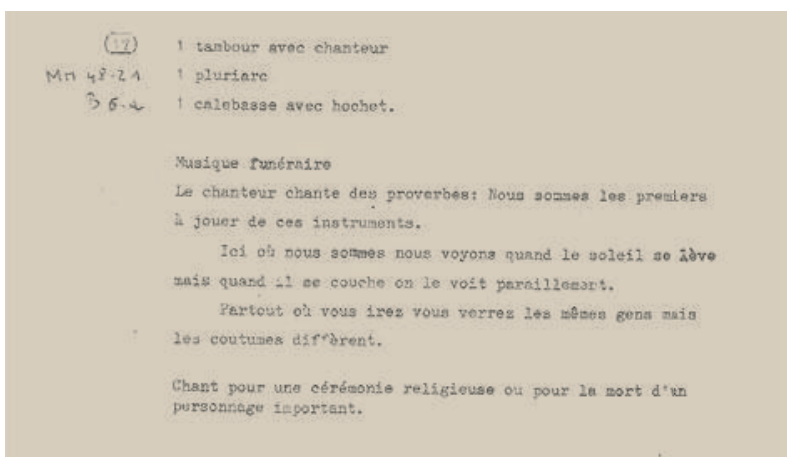

fig. 5 Face de disque 17,

Notes de terrain: 18
GKNDIKOLO, 29.8.46

Peterse

is poterie est faite par les fembes.

Après que la terre a b́té préparóe, lo poterio se monte svec deo boudins. Début de la poterie: D. 22 - $\mathbb{B}^{\circ} 9$ ot 10.

Quand lo pot est fait, on le décore de desains. La partie non décoréo est polie aveo un racloir de boiss D.22 - It० 8 .

L'Intérieur eat gratté avec un coquillage genre moule anis plus arrondi.

Lo pot eat aéché au soleil: D.25 - " ${ }^{0} 7$. Quelques jours (1 à 3 ?). Après quoi 11 est rentró dans in case cee la potièro oul 11 est exposb́, intérieur et extérieur, au á́obage da la funćo du fou, D.23 - No 5 .

Le pot après quelques joura de fumage dana la case est cuit de 2 s façon suivanto ; quelques pots sont disposés ensemble sur un 11t de branche sèches. Autour des pots et les recouvrant, on drease des branches qui forzent a la fols comas des wura et un toft. Per au bols. Cuisson de moins d'une dend-heure. Le pot eot terainé.

fig. 6 "Poterie»,

Notes de terrain: 67.

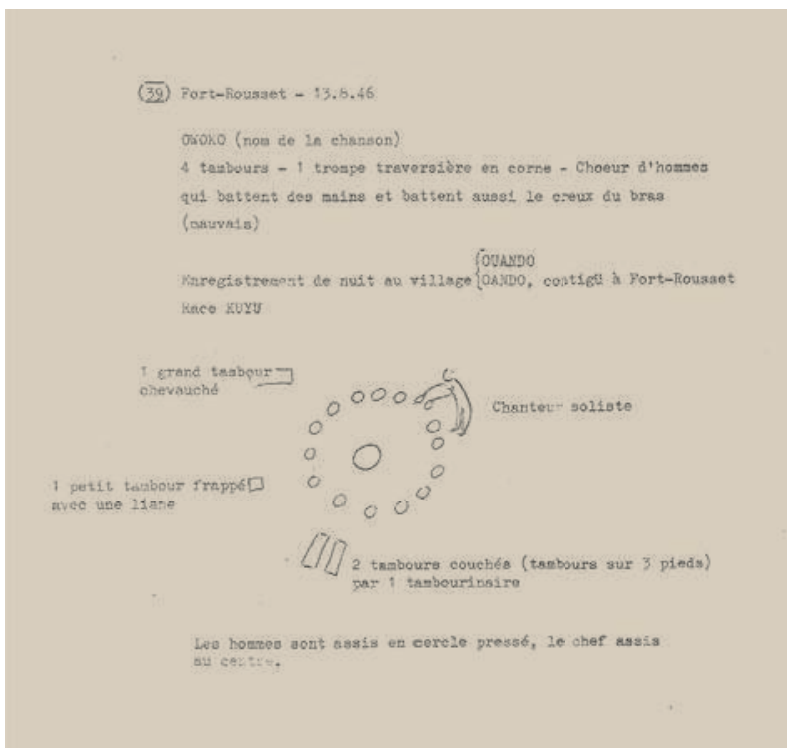

fig. 7 Face de disque 39,

Notes de terrain: 35. 
du texte (le même cahier commence par une note concernant une cithare sur bâton - okolobongo - portant la mention initiale «Fort-Rousset, 13.8.46", et indiquant plus bas que l'informateur, Victor Mohogo, est de race [et de langue] kouyou ${ }^{\mathbf{1 1}}$ ).

L'indication des instruments utilisés complète systématiquement ces éléments, comme l'illustre la note sur la face de disque 24 (fig. 4; Ewo était une ville du Congo ${ }^{12}$ ). Enfin, le sexe et le nombre d'exécutants sont indiqués de façon régulière:

[...] Danse de femmes - Histoire de coucheries - Se chante pour les jumeaux (naissance, mort), pour le mariage, etc. [...]
Les femmes dansent en cercle, à petits pas. Au centre les tambours joués par 3 jeunes gens ${ }^{13}$.

On retrouve un deuxième ensemble de renseignements attendus, mais de façon nettement moins systématique: le sujet et la fonction des musiques enregistrées (fig. 5), la langue et la position sociale des exécutants (par exemple, chef de village).

La vertu heuristique d'un modèle prescriptif réside par ailleurs dans le fait qu'une proportion importante des notes de terrain livre des renseignements inattendus au regard du modèle. Dans un premier ensemble de textes, Rouget réalise une ethnographie de la vie quotidienne des habitants rencontrés, parfois sans rapport avec la musique. Fin août 1946, il décrit par exemple les différentes phases de la fabrication d'une poterie (fig. 6; les références chiffrées renvoient à des photographies).

On peut lire dans le même sens les descriptions d'une chasse ou de la fabrication du vin de bambou, ou encore des éléments de recensement pour tel campement pygmée. D'autres notes décrivent des danses ou des cérémonies (déroulement, costumes...), comme la longue «Note sur la danse du okwi à Kongoboumba, du 15 novembre 1946 " :

Musiciens: 4 tambours par 3 tambourinaires. 1 tôle ondulée convexe, posée par terre, frappée par 3 hommes, tenant une baguette dans chaque main. Demi-cercle d'hommes jeunes tenant tous 2 baguettes de bambou qu'ils entrechoquent. Ce demi-cercle se tient à droite des tambourinaires. Chœur h.[ommes] et solo h. À son tour chaque homme se détache et vient danser un pas devant le tambour ${ }^{14}$. [...]

Un schéma illustre parfois ce type de description ou des fiches portant moins de renseignements (fig. 7). Quelques fiches, enfin, contiennent des éléments assez généraux d'analyse musicale au sens large de description du langage musical, comme cet extrait de l'évocation d'une «danse des enfants» (pygmées):

Les enfants battent des mains et poussent leurs yodlages ${ }^{\mathbf{1 5}}$, s'arrêtent, repartent, comme s'ils attendaient d'être en phase. Quand ça marche les vieux s'y mettent. D'une part en chant yodlé des enfants à plusieurs parties, avec les tambours qui battent en triolet, avec un système compliqué de temps forts, d'autre part les adultes qui chantent à la base une pédale faite d'une longue tenue qui baisse d'un ton (?) dans le cours de la tenue et s'évanouit. Cette pédale dure de 9 à 11 temps ${ }^{16}$ (?). 
On notera dans le même sens l'utilisation de termes techniques (ostinato, unisson...) dans de longues descriptions isolées ou dans des textes plus courts et directement associés à un enregistrement (par exemple: «Longue phrase soliste et longue réponse du chœur, avec chromatisme et dissonance $\left.{ }^{\mathbf{1 7}} »\right)$.

Le travail réalisé en mission a donc effectivement mis en pratique le programme défini en amont, mais en l'enrichissant par une observation diversifiée qui s'est très ponctuellement traduite par le recours à l'analyse musicale.

II faut par ailleurs s'interroger sur l'éventuelle spécificité du statut des informateurs dans l'ensemble du dispositif méthodologique de l'ethnographe: la dimension descriptive, qui domine largement dans le texte des notes de terrain, provient principalement de l'observation ethnographique, mais certains passages renvoient à cette autre source d'informations. L'auteur précise parfois le nom de l'informateur. Dans une fiche organologique concernant une cithare sur bâton, qui associe à un dessin des éléments de terminologie vernaculaire et des renseignements sur son usage, il précise par exemple que l'informateur est Victor Mohogo, de «race» et de langue kouyou ${ }^{18}$. Une fiche de renseignements sur le vin de bambou se présente de même comme une «interview avec Pierre Ambenga 19 ". Dans d'autres passages, le nom de l'informateur n'est pas indiqué: “L'instrument seul parlerait comme un homme. L'informateur (par le truchement d'un interprète), interrogé, répond qu'il chante les louanges d'une femme qu'il aime et que si celle-ci l'entendait sans le voir, elle comprendrait ${ }^{20}$. " II y a parfois, dans ce cas, plusieurs informateurs: «Les femmes dansent en cercle autour des musiciens groupés, dont le centre est le chanteur. Elles se suivent en file, avançant à petit pas en se déhanchant et en ondulant les épaules. Cette danse devrait, d'après les informateurs, s'exécuter avec hommes et femmes $\mathbf{2 1}$. 》

Les informateurs, qu'ils soient précisément nommés ou non, délivrent donc des renseignements dont le statut épistémologique est exactement le même que celui des éléments observés: ces informations rejoignent directement le corpus de connaissances descriptives et sont ainsi intégrées à une ethnographie globale qu'on peut effectivement appeler avec Rouget «substantielle», c'est-à-dire destinée à procurer de la matière ou du contenu. Inversement, les notes de terrain contiennent très peu de passages explicitant la nature des relations entre l'informateur et l'ethnographe, ou décrivant ce dernier lui-même en situation. On peut lire l'un des rares passages illustrant une telle réflexivité:

\footnotetext{
À la descente, au couchant. 5 ou 6 pirogues étaient ensemble. Voguant à la dérive. Un type à l'arrière d'une pirogue redressait vaguement de temps à autre. Le vieil Akili est venu nous rejoindre, tout tranquillement. À l'arrière les pirogues se sont égayées. On avait beaucoup bu. Les types disaient
}

12. Dans l'ensemble des Notes de terrain, des renseignements sur les instruments de musique (matériaux, décorations...) sont indiqués régulièrement et constituent parfois une fiche entière, comme semble le prescrire le modèle dans son quatrième point - mais sans aucune autre précision (fig. 2).

13. Notes de terrain: 43.

14. Ibid. : 285

15. La technique vocale du yodel repose sur l'alternance rapide entre voix de tête et voix de poitrine.

16. Notes de terrain: 69 (les points d'interrogation sont dans le texte).
18. Ibid. : 34

19. Ibid : 105

20. Ibid. : 35.

21. Ibid. : 63

22. Ibid. : 104 


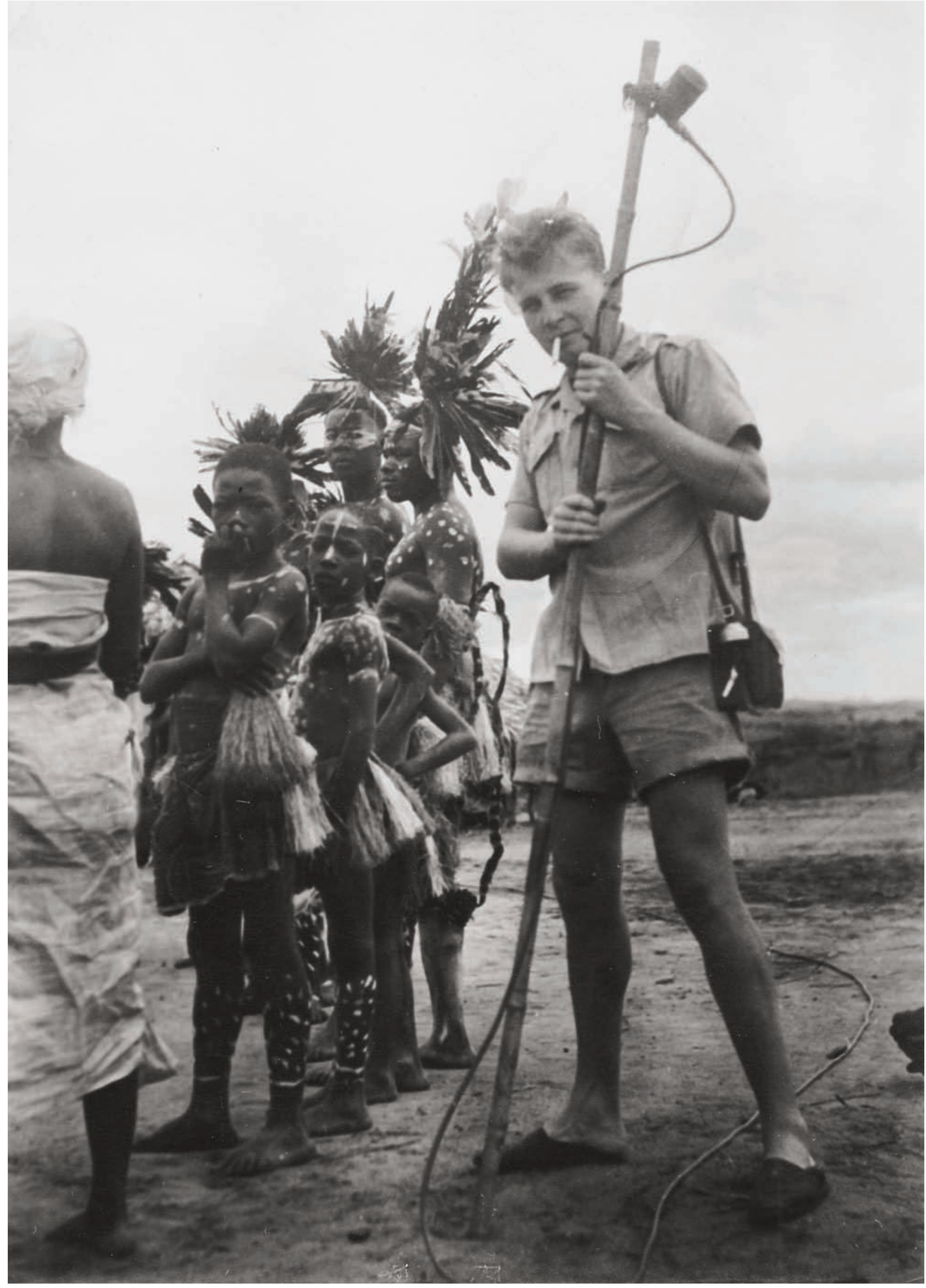


Rouget s'est donc attaché à décrire, à partir de l'observation directe et des propos des informateurs, un ensemble de réalités présentées comme objectives - à une époque où des mises en garde méthodologiques importantes avaient déjà été formulées à l'encontre du statut exclusif ou dominant de la description (voir par exemple Malinowski 1989 [1922]: 65 ou Mauss 2002 [1947]: 21-22). Son refus, aujourd'hui, de parler d'épistémologie à propos de la mission Ogooué-Congo est peut-être une façon de désigner le positivisme qui caractérisait cette entreprise, au contraire d'une problématisation qui aurait consisté à construire progressivement son objet d'étude - ce qu'il fera précisément dans La Musique et la Transe.

II est enfin nécessaire d'élargir la mise en contexte de cette ethnographie, réalisée au Moyen-Congo et au Gabon, pour la caractériser plus complètement. Certains travaux anglo-saxons proposent une réflexion sur les relations entre l'histoire de l'ethnomusicologie et celle des différentes formes de domination coloniale (Agawu 2003; Barz et Cooley 2008). Dans un esprit souvent différent, une bibliographie devenue relativement abondante éclaire les rapports entre l'ethnologie française et son contexte colonial (Sibeud 2002, 2006). L'absence de travaux comparables sur l'histoire de l'ethnomusicologie en France m'a incité à questionner Rouget sur son engagement politique à une époque où la France était déjà confrontée au début de la décolonisation dans certaines parties de son empire (Ageron 1994 ; Bénot 2003).

Rouget m'a expliqué qu'il était connu, en 1946, pour être communiste, alors que Jacques Dupont, le cinéaste de la mission qui a réalisé les films documentaires (Dupont 1946a, 1946b, 1946c), «était d'extrême droite (il fera partie de la Légion française à la guerre de Corée), fascisant, parfaitement colonialiste $\mathbf{2 3}$ ». II m'a également confié que «[son] communisme était surtout un communisme métropolitain. La décolonisation n'était pas alors le grand sujet, pas un sujet d'actualité politique ${ }^{\mathbf{2 4}}$. " Rouget, né en 1916, avait 20 ans à l'époque du Front populaire et 30 ans quand il a participé à la mission Ogooué-Congo: il appartient donc à une génération politique pour qui, sur le plan international, l'antifascisme a le plus souvent primé sur l'anticolonialisme. Mais il se souvient aujourd'hui qu'il partageait avec plusieurs membres de la mission une "vision très amicale de l'Afrique, pas du tout un esprit de colonisateur », et qu'ils furent dénoncés par des administrateurs pour avoir par exemple partagé des repas avec des Africains ${ }^{25}$.

Les réalités décrites en 1946 ont exclusivement concerné des musiques considérées comme éloignées des influences européennes et n'étant pas touchées par des phénomènes de métissage. On peut avancer l'hypothèse que de telles influences n'existaient pas alors, notamment en pays pygmée, mais il apparaît bien plus certainement que l'examen de cette question n'était pas envisagé en amont de l'enquête ${ }^{26}$. Rouget était communiste, éprouvait un sentiment de profonde sympathie à l'égard des populations rencontrées en Afrique, mais n'était pas radicalement anticolonialiste. Peut-être l'ethnomusicologue n'a-t-il pas considéré, au cours de la mission, la question coloniale comme importante: il ne se serait donc pas attaché à discerner dans ce qu'il voyait la traduction, au moins partielle, d'un rapport colonial. Peut-être au contraire a-t-il été beaucoup plus sensible à ce dernier
23. Entretien 31 mai 2008

24. Entretien, 21 septembre 2009

25. Entretien, 11 octobre 2009.

\section{ci-contre \\ fig. 8 \\ Noël Ballif, Membres de la Mission Ogooué-Congo. Noël Ballif au cours d'un enregistrement, 1946 (C) musée du quai Branly, photo Noël Ballif.}

\section{ci-dessous}

fig. 9

Noël Ballif, Danse des Bibaniki. Mission OgoouéCongo, 1946 @ ) musée du quai Branly, photo Noël Ballif.
26. Certains travaux ethnomusicologiques l'avaient déjà abordée explicitement, comme le livre publié en 1934 par Percival Kirby, consacré aux instruments de musique d'Afrique du Sud et dans lequel plusieurs passages importants sont consacrés aux instruments européens joués par les Africains (notamment Kirby 1968 [1934] : 257-259). Je remercie Emmanuelle Olivier d'avoir attiré mon attention sur ce point.

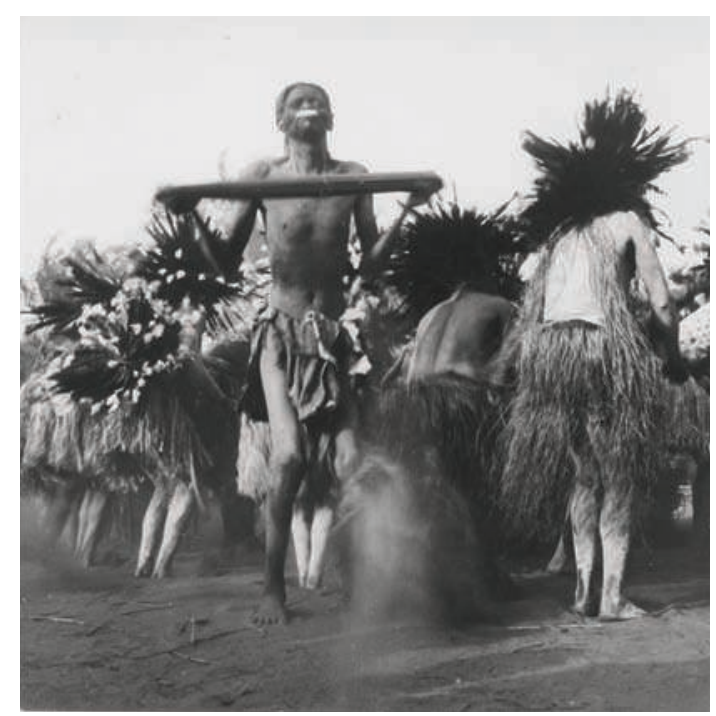




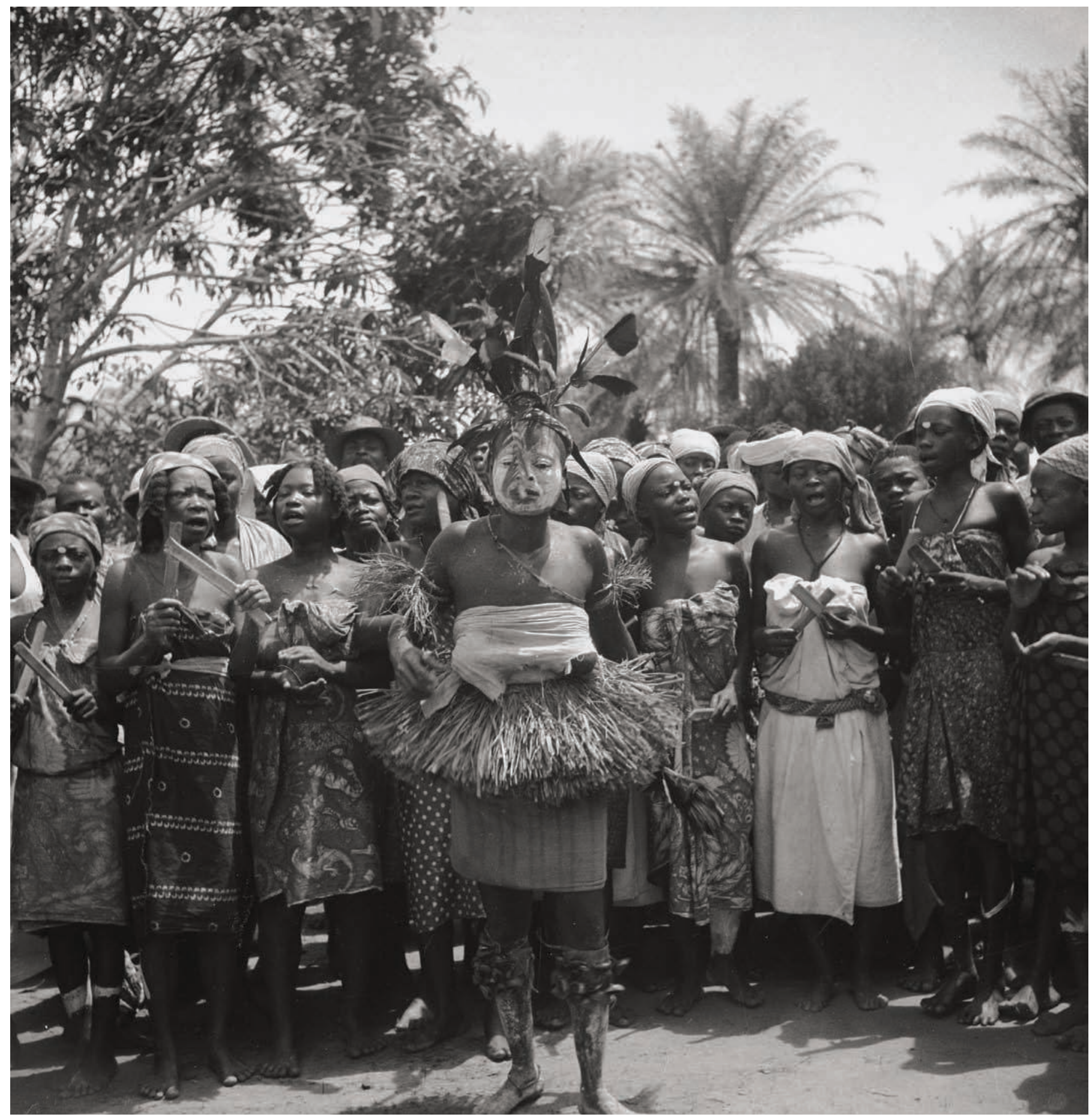

page 192 , ci-dessus

et page 215

fig. 10

Noël Ballif, Groupe de

femmes chantant.

Mission Ogooué-Congo, 1946.

Don de Florence Ballif N'Diaye

(C) musée du quai Branly,

photo Noël Ballif. 
et a-t-il volontairement, de façon presque militante, laissé de côté la question des influences européennes dans l'optique de ce qu'il appellera plus tard une ethnomusicologie d'urgence, destinée à accorder la priorité à la sauvegarde de cultures menacées de disparition par l'occidentalisation. Quelle que soit I'hypothèse retenue, il apparaît bien que Rouget s'est alors concentré exclusivement sur la description d'un ensemble de traits culturels supposés traditionnels, en écartant l'historicité des faits observés et la question d'éventuelles influences européennes, passées ou présentes.

On peut se demander si cette exclusivité n'illustre pas plus généralement ce que Jean-Loup Amselle a appelé la raison ethnologique, une "démarche discontinuiste qui consiste à extraire, purifier et classer afin de dégager des types » et qui s'oppose à la logique métisse, «c'est-à-dire une approche continuiste qui à l'inverse mettrait l'accent sur l'indistinction ou le syncrétisme originaire" (Amselle 1999: 9-10). Les notes de terrain de la mission Ogooué-Congo, par exemple, semblent illustrer cette raison ethnologique par leur usage géographique et classificatoire de la race, un des éléments qu'il fallait renseigner systématiquement sur le terrain pour pouvoir ordonner la réalité observée, au risque d'entériner l'«invention des ethnies » réalisée par les administrateurs coloniaux (voir sur ce thème ibid: : 22-28, qui prolonge le livre classique publié en 1985 par le même auteur et Elikia M'Bokolo sous le titre Au cœur de l'ethnie. Ethnies, tribalisme et État en Afrique).

Ces quelques éléments incitent du moins à rechercher en dehors de l'ethnographie proprement dite la spécificité du travail réalisé en mission. 
27. À titre d'exemple, la face B1a (première face du premier disque de 1948) est issue de l'« original n० 526 ".

28. On trouve régulièrement, dans les Notes de terrain, une brève indication sur la qualité sonore d'une séquence enregistrée, par exemple "mauvais" ou «le meilleur» (Notes de terrain: 170).

29. Notes de terrain : 61.

30. Ibid. : 62

31. Notes sur les disques: B11a.

32. Ibid. : B1a.

\section{Épistémologie et enregistrements sonores}

Dans leur introduction au numéro de L'Homme consacré à «Musique et anthropologie», Bernard Lortat-Jacob et Miriam Rovsing Olsen, pour illustrer l'importance des technologies successives d'enregistrement sonore dans l'histoire de l'ethnomusicologie, citaient Béla Bartók, qui écrivait en 1937: «Je le dis sans hésiter, la science du folklore musical (alias ethnomusicologie) doit son développement actuel à Edison. »(Lortat-Jacob et Rovsing Olsen 2004: 10, note 10) L'importance des enregistrements rapportés de la mission Ogooué-Congo encourage précisément à s'interroger sur la spécificité de leur statut épistémologique.

Pour comprendre la façon dont le matériau ethnographique collecté en mission a été ultérieurement exploité par Rouget pour commenter ces enregistrements sonores, il faut partir à nouveau d'un document inédit, un volume dactylographié de 92 pages longtemps conservé au département d'Ethnomusicologie du musée de l'Homme et intitulé Notes sur les disques de la mission Ogooué-Congo.

Ces Notes concernent une série éditée par le musée de l'Homme en 50 exemplaires hors commerce de 34 disques 78 tours pressés en 1948 à partir de certains enregistrements réalisés en mission deux ans plus tôt. Leur lecture et leur analyse peuvent heureusement s'appuyer sur un ensemble de renvois réciproques avec les notes de terrain prises en 1946: pour chaque face d'un disque de 1948, les Notes sur les disques précisent la face correspondante du disque gravé en mission ${ }^{\mathbf{2 7}}$. Inversement, des mentions manuscrites ont été portées a posteriori sur certaines Notes de terrain (voir fig. 5) pour renvoyer aux enregistrements de 1948. La consultation des deux documents permet ainsi de repérer et de comprendre dans le détail la filiation entre les deux types de notes. Elle éclaire la façon dont un certain nombre de renseignements ont été réunis en vue d'élaborer telle note sur un disque en particulier.

Une confrontation attentive permet premièrement de constater qu'une note sur une face de disque de 1948 est le résultat d'une sélection et d'une réunion de renseignements donnés dans plusieurs notes prises en 1946 (alors que la face de disque éditée en 1948 correspond nécessairement à un seul enregistrement sonore en mission). Cette pluralité de sources textuelles s'explique par le fait que plusieurs prises de son étaient réalisées en mission dans un même village, devant les musiciens, et que les renseignements notés successivement pouvaient être ensuite mobilisés pour commenter un seul enregistrement, jugé le meilleur 28.

Une illustration en est livrée par la face de disque B11a, issue de l'enregistrement 91 des Notes de terrain. Pour comprendre la genèse de la note de 1948, il faut lire les trois pages constituées par les notes prises à Ouesso le 23 août 1946. Le texte de la première page, non directement associé à un enregistrement, présente un orchestre en indiquant des éléments de terminologie vernaculaire et surtout des précisions organologiques: 
Joueurs de sanza: Calebasse tenue par une lanière autour du cou - la sanza est plongée dans la calebasse.

Sanza de 12 lames de fer. Les 2 sanza sont accordées à l'unisson. [...] Hochet en vannerie, contenant

La page suivante, après quelques autres précisions, porte les quatre premiers numéros des faces de disque gravées le même jour:

Orchestre ci-dessus avec chanteur soliste homme, chœur de femmes et d'hommes. Idem: Lisala: perroquet Le perroquet avait deux femmes. II laissait toujours la $2^{\mathrm{e}}$ à la maison et des coques, à manche de bois.

Nom: madjé (en bugõgo)

Les coques sont des noyaux de fruits.

Les bâtons entrechoqués: gada (en bugõgo ${ }^{29}$ ).

La dernière page de cet ensemble porte le $n^{\circ} 94$, dont le début indique le titre et le sujet du chant, ainsi que des précisions sur la façon de chanter.

L'ensemble de ces notes de terrain, associées à cinq enregistrements différents, constitue la source du commentaire de la face de disque B11a gravée en 1948, transcrit ici en intégralité:

MH 48-11 (original $n^{\circ}$ 91) B11a «Lisala». Lisala: perroquet. La chanson est une fable: le perroquet avait deux femmes, il laissait toujours la seconde à la maison et emmenait la première à la fête.

Autour des musiciens les femmes forment un cercle et, les unes derrière les autres, tournent lentement, avançant à petits pas en se déhanchant et en roulant les épaules.

Le chanteur soliste agite un hochet fait d'une baguette de bois servant de manche, terminée par une boule de emmenait la $1^{\text {re }}$ à la fête. [Une mention manuscrite porte ici dans la marge « $\mathrm{MH}$ 48-11 B11a», référence du disque édité deux ans plus tard ${ }^{30}$.] [...]

Ces opérations de sélection et de réunion des informations ne suffisent cependant pas à rendre compte du travail de composition qui aboutit aux commentaires de 1948. Il faut également souligner que ceux-ci aboutissent systématiquement à une contextualisation du même type: ils adoptent tous la même structure ternaire, illustrée par l'exemple précédent, en présentant successivement le sujet et la fonction de la musique, la disposition des musiciens et des danseurs (nombre d'exécutants et d'instruments, déplacements...), puis des précisions organologiques. La progression se traduit ainsi par deux changements successifs de focale, mais ces trois parties peuvent aussi se retrouver dans un autre ordre, comme dans la première note du volume:

\section{[...] Mbango se désole parce que} son couteau est resté à l'autre bout du village. Instruments:

$1^{\circ}$. Trois tambours battus chacun par un homme. Tambours à deux peaux. [...] Les hommes forment un demi-cercle, les femmes le demi-cercle complémentaire qui lui fait face; les tambours sont disposés à côté les uns des autres, sur le bord du cercle et séparent les hommes et les femmes. Danse sur place ${ }^{32}$.

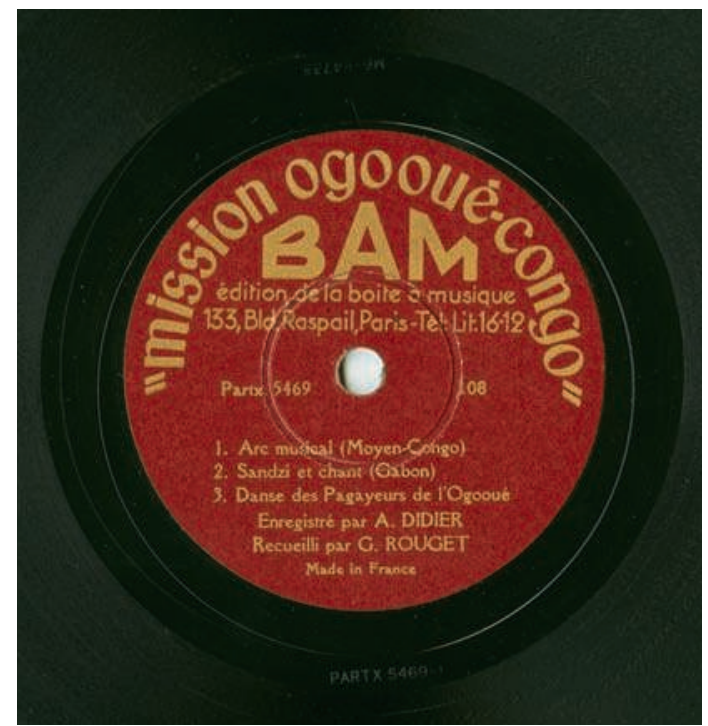

fig. 11

Mission Ogooué-Congo, édition de la boîte à musique (BAM), 78 T, 1946. Collection Bibliothèque nationale de France.

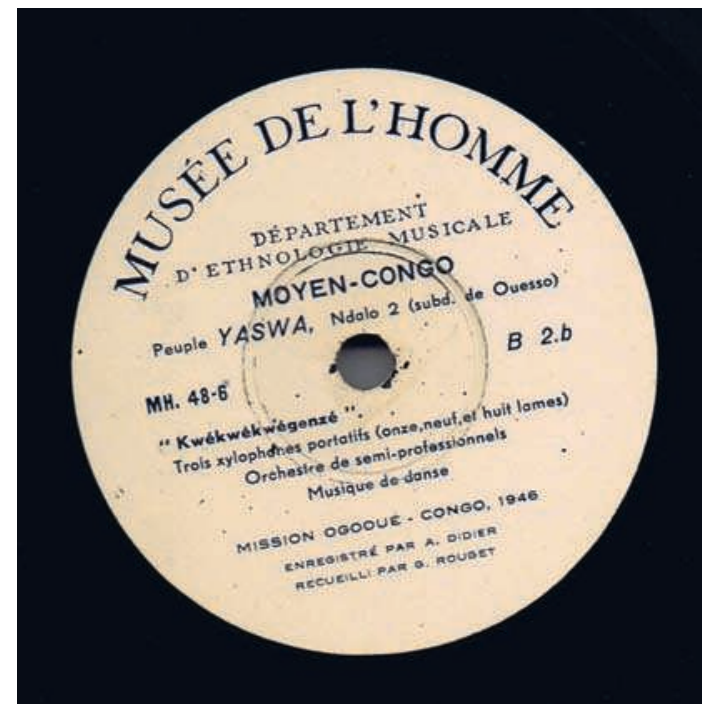

fig. 12

Rondelle du disque

"KWEKWEKWEGENZE,

Trois xylophones portatifs (onze, neuf et huit lames) ". Enregistrements sonores collectés en 1946 lors de la mission Ogooué-Congo par Gilbert Rouget, Pierre Gaisseau et André Didier. Archives sonores

CNRS - Musée de l'Homme. Centre de Recherche en Ethnomusicologie. 
33. Ibid. : B5a.

34. Ibid. : B11a.

35. Plusieurs références discographiques sont indiquées en fin d'article.
II faut enfin noter l'absence complète, dans les Notes sur les disques, d'éléments d'analyse musicale. Une lecture exhaustive révèle que seuls deux passages s'arrêtent sur le seuil d'une telle analyse en évoquant très ponctuellement une donnée qui pourrait introduire une étude de la systématique musicale. Rouget présente un orchestre de cinq trompes d'ivoire et précise: «Chaque trompe ne donne qu'un seul son et la musique résulte de la mosaïque de ces cinq sons ${ }^{33}$. . Par ailleurs, dans un exemple précédent, il note que les deux sanza, «à douze lames métalliques, sont accordées à l'unisson l'une de l'autre ${ }^{\mathbf{3 4}}$ ». Ces allusions à des hauteurs de sons sont directement liées à des précisions organologiques et on chercherait en vain dans le reste du volume le compte rendu précis de telle structure, rythmique ou mélodique par exemple. L'apparition, même ponctuelle, d'éléments d'analyse musicale dans les Notes de terrain de 1946 incite à considérer cette absence comme significative et résultant d'un choix. Elle confirme par défaut le statut exclusif, dans les Notes de 1948, de la structure ternaire déjà évoquée (sujet et fonction de la musique, description de la performance et précisions organologiques).

Cette structure se retrouve bien au-delà d'un seul document inédit. Les enregistrements de la mission Ogooué-Congo ont donné naissance à plusieurs disques, gravés et édités à des dates variables ${ }^{35}$. Parmi différents exemples, un disque 33 tours intitulé Musique bantou d'Afrique équatoriale (Rouget 1958) contient une notice de quatre pages signée par Rouget. Après une dizaine de lignes définissant "Bantou» et localisant les peuples qui se rattachent à cette "grande famille linguistique", l'auteur présente successivement chacune des plages en un texte de longueur variable (de 6 à 19 lignes). Ces commentaires sont organisés exactement de la même façon que les Notes sur les disques de 1948: les trois éléments de la même structure ternaire sont parfois présentés dans un ordre différent (par exemple, les positions et les déplacements des instrumentistes et des danseurs peuvent être signalés en fin de texte), mais leur association systématique est au principe de chacun des textes et constitue donc un socle épistémologique, c'est-à-dire le schème fondamental et récurrent de présentation des réalités musicales inscrites dans leur contexte et observées sur le terrain. Cette récurrence est d'autant plus significative que Rouget n'a pas rédigé de monographie issue de son travail en mission, genre classique pour l'époque, ce qui fait des notices des différents disques édités les principaux objets textuels en relation directe avec la mission Ogooué-Congo - j'évoquerai plus loin le caractère particulier de l'article consacré aux Pygmées en 2004.

Comme sans doute toutes les notices de disque, qui ne sont pas des textes autonomes, celles écrites par Rouget à partir de son expérience ethnographique semblent d'abord principalement destinées à guider l'écoute. Par ailleurs, leur statut possède une relative spécificité: elles se trouvent dans un rapport de complémentarité, mais aussi de franche altérité avec les enregistrements correspondants, au contraire de la synchronie qui associe étroitement le déroulement des images et leur commentaire dans un film ethnographique. Le temps de la lecture ne saurait s'adapter au temps linéaire de l'écoute. Mais une spécificité plus grande, dans le contexte ethnologique, réside ici dans le fait de rassembler de nombreuses infor- 


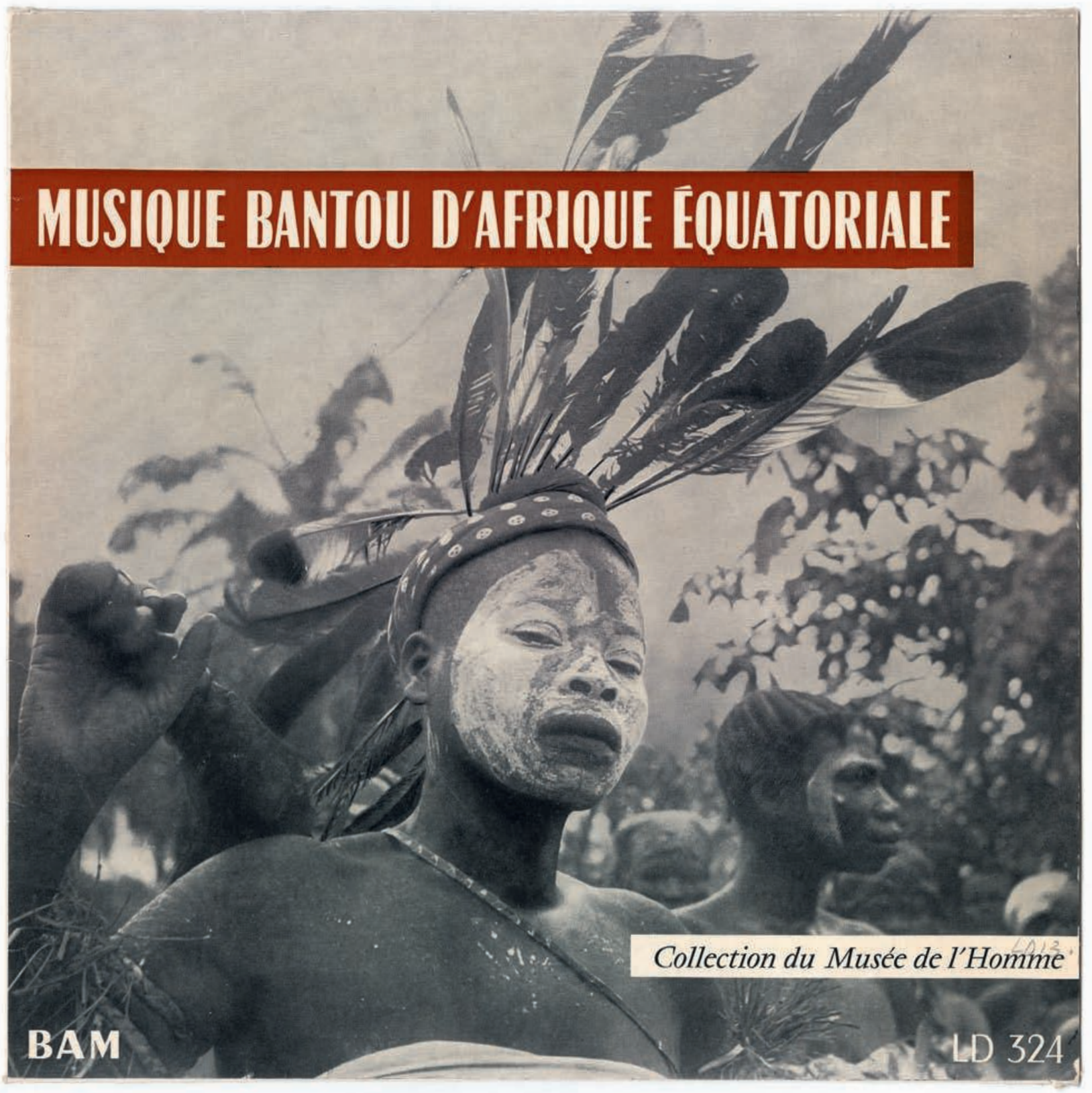

fig. 13

Pochette du disque (recto) Musique bantou d'Afrique

Equatoriale française, 1958 Enregistrements sonores collectés en 1946 lors de la mission Ogooué-Congo par Gilbert Rouget, Pierre Gaisseau et André Didier. Photo : Noël Ballif. Archives sonores CNRS -

Musée de l'Homme. Centre de

Recherche en Ethnomusicologie. 


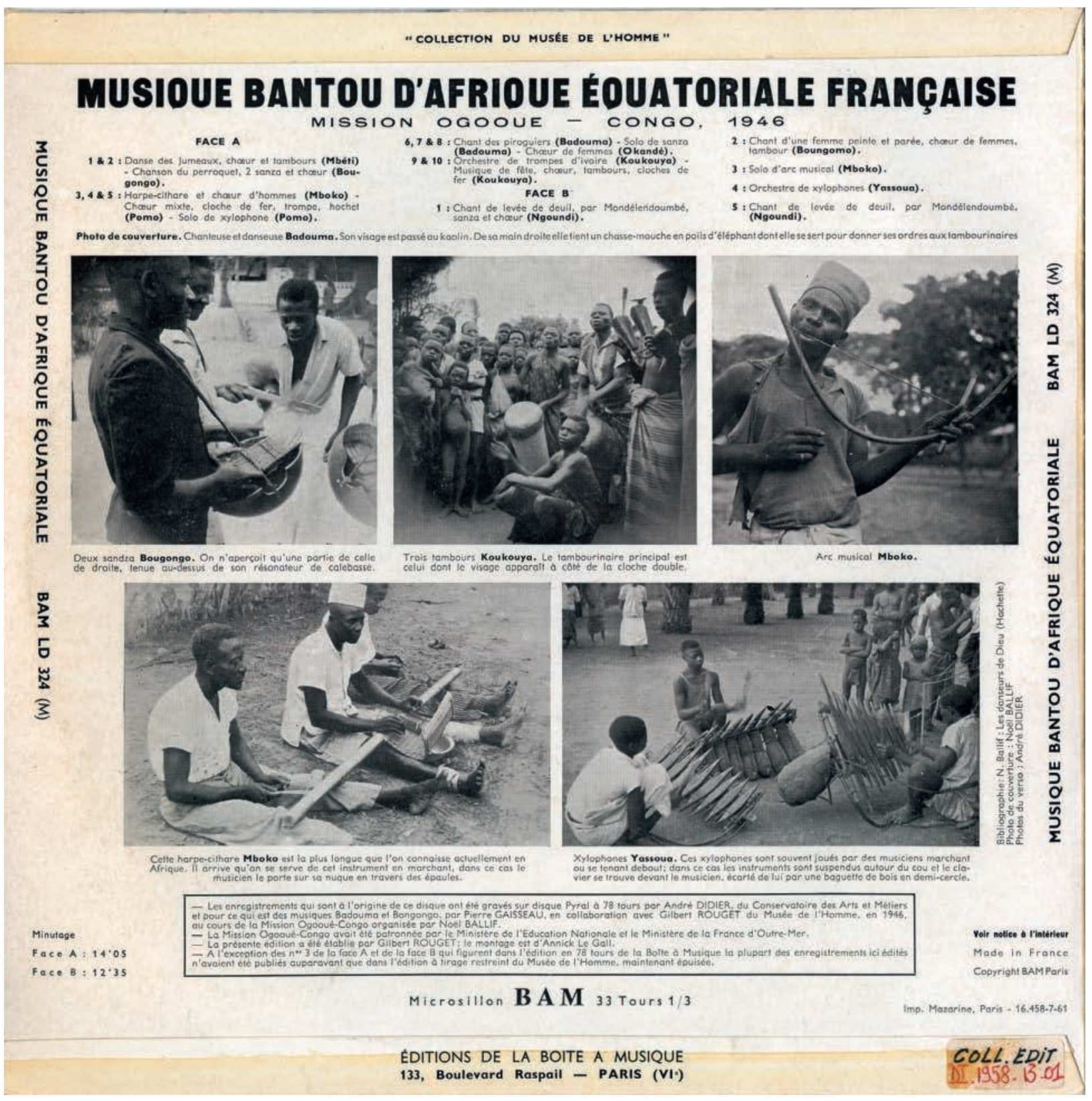

fig. 14

Pochette du disque (verso) Musique bantou d'Afrique Equatoriale

française, 1958. Enregistrements sonores collectés en 1946

lors de la mission Ogooué-Congo par Gilbert Rouget, Pierre Gaisseau et André Didier. Photos: André Didier. Textes : Gilbert Rouget. Archives sonores CNRS -

musée de l'Homme. Centre de

Recherche en Ethnomusicologie. 
mations dans un format court et un genre très homogène, ce qui semble favoriser, parmi d'autres facteurs, l'absence de vigilance épistémologique quant au statut des informateurs et de leur parole, complètement fondue dans le matériau descriptif.

Plus globalement, le disque pris dans son ensemble se caractérise donc par une dualité fortement contrastée entre la notice et l'enregistrement lui-même, dont il faut pour finir analyser le statut. C'est en 1932 qu'est inventé le type de disque souple, à gravure directe, qui sera utilisé pendant la mission de 1946 (sur l'histoire de l'enregistrement sonore, voir Tournès 2006, 2008; Sterne 2003). Ce disque «Pyral », du nom de la société Pyrolac qui en fournissait le matériau, fut notamment utilisé en 1939 par Claudie Marcel-Dubois pendant la mission de folklore musical en Basse-Bretagne qui dépendait du musée national des Arts et Traditions populaires et permit de rapporter 93 disques gravés sur le terrain, représentant sept heures d'enregistrements (Le Gonidec [éd.] 2009). Sept ans plus tard, sur les conseils de Schaeffner, Rouget se rendit au Conservatoire national des arts et métiers et y rencontra André Didier, alors assistant et spécialiste des techniques de l'enregistrement du son et de l'image, qui lui proposa de se joindre aux membres de la mission Ogooué-Congo ${ }^{36}$. Les enregistrements sonores réalisés par les deux hommes sur le terrain - Didier fut remplacé par Pierre-Dominique Gaisseau dans la seconde partie de la mission, lors de la descente de l'Ogooué - devaient représenter quelque six cents faces de disque, soit environ vingt-cinq heures de musique.

Ce disque Pyral était constitué d'une plaque métallique ronde en aluminium recouverte d'une couche d'acétate de cellulose épaisse, permettant de graver un sillon sans atteindre le métal. Son diamètre $(30 \mathrm{~cm})$ et sa vitesse de rotation (78 tours par minute) limitaient sa durée à 4 minutes sur chaque face, mais l'innovation technologique majeure par rapport au cylindre consistait à pouvoir faire une prise directe et lire l'enregistrement immédiatement après la gravure, annonçant l'usage postérieur du magnétophone qui se diffusera largement dans les années 1950.

Une première implication de ce dispositif technique réside dans la possibilité d'évaluer et de noter immédiatement sur le terrain la qualité des différentes prises, en accumulant ainsi des indications écrites qui détermineront directement la sélection opérée en vue de réaliser et d'éditer ultérieurement des disques. Les Notes de terrain contiennent un très grand nombre de ces commentaires, plus ou moins brefs, tels qu'ils apparaissent dans un passage reproduit plus haut (fig. 4: «Idem - meilleur. »). L'ensemble participe donc d'un renversement méthodologique au terme duquel les notes prises sur le terrain permettent en partie de constituer la documentation sonore - au contraire, par exemple, de la mission Dakar-Djibouti, dont les notes de terrain rédigées par Schaeffner contiennent quelques renvois aux cylindres enregistrés qui apparaissent clairement comme les illustrations sonores et très ponctuelles du texte (Gérard 2009).

Une deuxième implication se traduit par la recherche manifeste d'un effet de réel au fil des différentes prises: on devine à la lecture des Notes de terrain que Rouget s'est fondé sur l'écoute immédiate de chaque
36. Entretien, 15 décembre 2009 
prise pour gagner en fidélité à l'égard des musiques enregistrées et écarter ce qu'il percevait comme des sons inutiles ou des bruits parasites:

Chœur de jeunes (h. et f.) avec 3 tambours battus baguettes. enregistrement de loin. Grand désordre, conversations près du micro ${ }^{37}$.

L'usage d'une nouvelle technique a incontestablement permis de mieux circonscrire et restituer l'objet musical, et d'aboutir ainsi à des enregistrements d'une grande qualité, mais on peut penser aussi qu'il a indirectement contribué à modifier ou redéfinir le statut de la réalité dans l'enquête ethnographique globale. L'enrichissement méthodologique constitué par la recherche, à travers le son, d'une authenticité ou d'une véracité a peut-être mis en place une forme de concurrence épistémologique avec d'autres accès à la vérité. II a pu favoriser par exemple, dans les différentes étapes du dispositif, l'intégration exclusive de la parole des informateurs à l'ensemble des données descriptives et destinées à commenter les enregistrements.

Une troisième implication s'explique enfin par la présence, dans les Notes de terrain, de jugements qui ne concernent pas la qualité de telle prise de son mais celle de la musique elle-même:

Chœur h. chœur f. Tambours.

Battements de mains. à l'arrêt sur cri

d'ensemble, reprise immédiate.

À 3' très bon HEHA d'une femme puis
HEA de femmes et d'hommes. Battements de mains violents. Très bon. Un peu confus aux HEHA. Le meilleur comme musique ${ }^{38}$.

On peut lire, pour d'autres faces de disque: «Moins bon. Moins bien rythmé» (p. 146) ou «Très beau» (p. 233). Ces jugements incitent à s'interroger sur le statut de l'expérience esthétique dans le protocole ethnographique. Un ethnographe constitue lui-même sa propre documentation (Mauss 2002 [1947]: 19-20), mais une spécificité relative réside peut-être ici dans le fait que le jugement esthétique participe, en orientant la sélection ultérieure des enregistrements retenus parmi les nombreuses prises, de la constitution même, au sens fort de fabrication, des archives sonores. Paradoxalement, l'innovation technologique permet à l'expérience esthétique, certainement présente dans toute anthropologie de la culture, de se déployer et de s'intégrer, plus qu'auparavant, à certaines étapes préliminaires et fondatrices du parcours scientifique. L'exploitation des cylindres enregistrés dans les années 1930, dans les phases suivantes du travail ethnomusicologique, était sans doute indissociable de tels jugements, mais ceux-ci n'avaient pas aussi directement déterminé le contenu même des cylindres.

Le statut particulier des enregistrements sonores permet donc bien de circonscrire un ensemble d'implications spécifiques qui permettent d'évaluer plus généralement l'importance de la mission Ogooué-Congo.

La première mission de Rouget se caractérise, au terme de l'analyse, par une forme de modernité paradoxale qui réside dans l'articulation entre le positivisme d'une ethnographie classique, lui-même déterminé en partie par le contexte scientifique et plus global de l'immédiat après-guerre, et la vertu souvent heuristique de l'utilisation des techniques récentes d'enregistrement sonore. Cette modernité explique sans doute l'importance de la mission Ogooué-Congo dans la carrière ultérieure de l'ethnomusi- 
cologue, qui a ensuite travaillé de façon continue au sein du département d'Ethnomusicologie du musée de l'Homme, dont il a pris lui-même la direction à la suite de Schaeffner de 1965 jusqu'à sa retraite en 1985.

Rouget a effectué dans ce cadre vingt missions, dont dix-sept en Afrique et principalement au Bénin, qui lui ont permis d'éditer dans l'ensemble plus de soixante disques ${ }^{39}$. II a par ailleurs créé un studio d'enregistrement du son au musée de l'Homme en 1947, afin d'éditer de manière autonome des disques issus d'enregistrements recueillis sur le terrain, à commencer par ceux réalisés l'année précédente. II a fondé par la suite avec Jean Rouch, en 1964, le laboratoire audiovisuel de l'École pratique des hautes études ( $\vee$ e section), puis créé au sein du département d'Ethnomusicologie, quatre ans plus tard, une formation de recherche du CNRS incluant un laboratoire d'enregistrement, d'analyse et de transcription automatique du son géré par un ingénieur et consacré en priorité aux archives sonores du département. Ces dispositifs institutionnels sont eux-mêmes inséparables de certains travaux scientifiques, notamment dans le domaine de l'analyse musicale: Rouget, dans un article devenu classique (Rouget 1970), a par exemple présenté de façon novatrice l'analyse comparée de deux chants, africain et fuégien, en recourant à différentes représentations de la musique (sonagrammes et transcriptions solfégiques), dont certaines étaient rendues possibles par l'analyse du son (pour une réflexion collective et plus large sur les représentations de la musique en ethnomusicologie et à partir d'enregistrements sonores, voir Rouget 1981). II a enfin publié plus récemment dans L'Homme un article qui s'appuie en partie sur la documentation rassemblée en 1946 chez les Pygmées. Celui-ci permet notamment au lecteur de voir des photographies prises pendant ce séjour (planches horstexte) et d'entendre des extraits de la musique enregistrée (plages 1 à 4 du CD encarté [Rouget 2004]).

Il apparaît donc rétrospectivement que la mission Ogooué-Congo a représenté pour Rouget le premier moment d'une longue contribution offerte à l'appropriation collective des techniques successives d'enregistrement sonore, certainement la plus importante dans I'histoire de l'ethnomusicologie en France.

Mais I'historien d'une discipline est soucieux de comprendre plus globalement dans quelle mesure l'institutionnalisation d'un domaine de savoir s'est accompagnée d'une structuration épistémologique: si l'on reste attentif aux caractéristiques de l'ethnographie réalisée en 1946, l'exemple ponctuel de la mission Ogooué-Congo incite à situer certaines spécificités liées à l'enregistrement sonore dans le contexte d'une indiscernabilité globale entre l'ethnomusicologie naissante et l'ethnologie de l'époque. Mes recherches en cours, destinées à analyser d'autres missions ou des travaux ethnomusicologiques de nature différente, devraient donc permettre de proposer les résultats d'une enquête élargie, précisant cette impression plus générale: une discipline semble être souvent plus qu'une réalité institutionnelle et moins qu'une épistémologie distincte.

\section{Notes de terrain : 149}

38. Ibid. : 166

39. L'intégralité de cette documentation musicale est aujourd'hui numérisée et représente trois cent cinquante $C D$. 


\section{Rouget, Gilbert}

1948a Musiques pygmées et nègres d'Afrique équatoriale française. Mission Ogooué-Congo 1946, longue notice illustrée de Gilbert Rouget, 3 disques 78 tours. Paris, Boîte à Musique.

1948b Moyen-Congo et Gabon. Mission Ogooué-Congo, 34 disques 78 tours. Paris, collection musée de l'Homme (hors commerce).

1949 Music of Equatorial Africa. Mission Ogooué-Congo, notices de Gilbert Rouget, 4 disques 78 tours. New York, Ethnic Folkways.

1958 Musique bantou d'Afrique équatoriale, disque 33 tours. Paris, Boîte à Musique.

1959 Musique pygmée d'Afrique équatoriale, disque 33 tours. Paris, Boîte à Musique.

\section{Dupont, Jacques}

1946a Au pays des Pygmées, Atlantic Film (22 mn).

1946b Pirogues sur l'Ogooué, Atlantic Film (26 mn).

1946c Danses congolaises, Atlantic Film (11 mn).

\section{Jourdain, Stéphane}

2008 Gilbert Rouget, un parcours ethnologique, Bernard Lortat-Jacob et Claude Kiejman (entretien), DVD. Paris, La Huit Production ("L'ethnologie en héritage » 3).

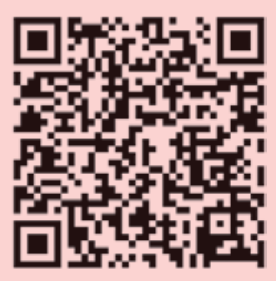

Ce code donne accès aux archives sonores CNRS - musée de l'Homme,

Centre de recherche

en ethnomusicologie (CREM, LESC-UMR 7186

CNRS-université Paris Ouest). Consultation libre:

http://archives.crem-cnrs. fr/archives/collections/ CNRSMH_E 1958013 001/.

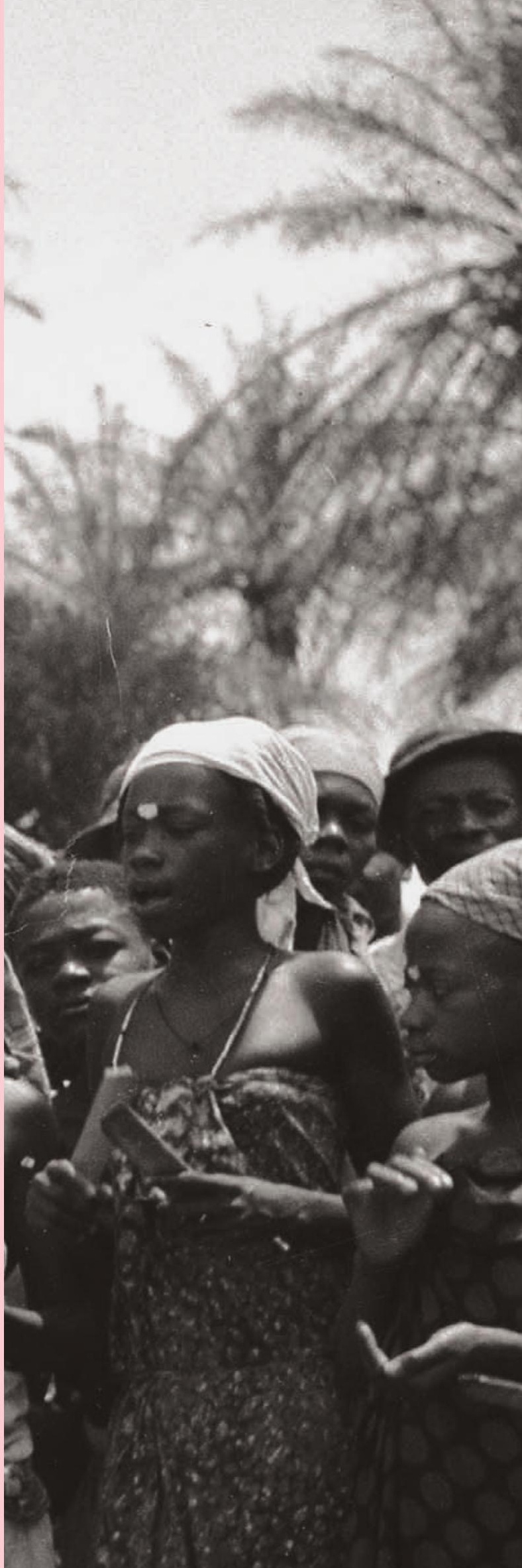

